\title{
Electrostatic and scalar fields
}

\author{
Adam Noble and Dino A. Jaroszynski \\ SUPA Department of Physics, University of Strathclyde, Glasgow, Scotland, UK
}

\begin{abstract}
The theory of electrostatics is embedded within Maxwell's electrodynamics, which is one of the most successful physical theories ever produced. However, a more direct generalisation of electrostatics to make it compatible with relativity would have been to replace it with the sourced massless Klein-Gordon equation. This paper gains insight into these two theories by comparing their structures and consequences in the static limit, finding that the two agree in terms of the field dynamics, but differ substantially in terms of how charged particles interact with the fields. These differences are most prominent for ultra-relativistic particles interacting with strong fields, conditions relevant to the new generation of high power laser facilities. Further connections between electrodynamics and scalar dynamics are explored by unifying the theories in higher dimensional spacetime, analogously to the Kaluza-Klein unification of electrodynamics and gravity.
\end{abstract}

Keywords: Electrostatics, relativistic scalar fields, extra dimensions

\section{INTRODUCTION}

The nonrelativistic descriptions of electrostatic and gravitational interactions bear more than a passing resemblance to each other. Indeed, each can be described by a scalar field satisfying Poisson's equation, and the key differences (that electrical forces can be attractive or repulsive, while gravity is always attractive; and that a particle's 'gravitational charge' is proportional to its mass, while electric charge is independent) could be seen as coincidental. These differences became central to how the theories developed to incorporate relativity, into Maxwell's theory of electrodynamics*, and Einstein's general theory of relativity. It is worth noting that the straightforward relativistic generalisation of Poisson's equation to a (spacetime) scalar wave equation does not describe either electrodynamics or gravity, which instead are described by 4-vector and 4-tensor interactions, respectively (the latter corresponding to the geometry of spacetime).

Despite these differences, relativistic electrodynamics and gravitation remain closely related. A powerful illustration of this is the observation of Kaluza, ${ }^{1}$ that Einstein's equations in $5 \mathrm{D}$ contain not only their 4D restriction, but also Maxwell's equations. Similarly, though less well known, Maxwell's equations in 5D contain not only $4 \mathrm{D}$ electrodynamics, but also the equation for a relativistic scalar field (see Appendix A).

At a fundamental level, scalar fields are not plentiful. Indeed, classical physics is fully described by electrodynamics and gravity. Of the numerous additional fields introduced by the standard model of particle physics, only one, the Higgs field, is scalar. Nevertheless, extensions of the standard model almost inevitably produce a plethora of scalar fields. Moreover, in many cases of practical importance it is convenient to approximate more intricate interactions with simplified scalar versions. For example, the bulk behaviour of electrons in a laser pulse may be obtained by averaging out the rapid oscillations, replacing the (vector) Lorentz force with the ponderomotive potential, ${ }^{2}$ a scalar field determined by the laser intensity.

The goal of this paper is to compare the physics of electrostatic fields with their scalar equivalents. The motivation for this is twofold: firstly, to understand the dynamics of systems that can be described (fundamentally or as an approximation) by scalar fields. And perhaps more importantly, by analysing their differences from scalar interactions, to better understand features of electrostatic interactions, especially for ultra-relativistic particles interacting with very strong fields. Such conditions are relevant to upcoming experiments at new high

E-mail: adam.noble@strath.ac.uk

*Although Maxwell's theory preceded the development of special relativity, it is fully consistent with it, and was the primary influence on the formulation of the latter. 
power laser facilities, and though the fields produced at these facilities are anything but static, the present work lays the foundations for a more general study.

The rest of the paper is organised as follows. In Section 2, we explore the basic field equations, and find relations between scalar and electrostatic fields. In Section 3, we explore the motion of particles interacting with these fields, and how these draw out greater differences between the two cases. Conclusions are drawn in Section 4, while Appendix A demonstrates how electrodynamics and scalar fields can be unified in 5 spacetime dimensions.

Throughout, we employ the Einstein summation convention, taking Greek indices to range over $\{0,1,2,3\}$, with 0 timelike and $\{1,2,3\}$ spacelike (alternative indices used in the Appendix are defined in context). The metric tensor has signature +--- , so that timelike vectors square to positive values, and spacelike vectors to negative values. We work in Heaviside-Lorentz units, with $c=\epsilon_{0}=1$.

\section{FIELD DYNAMICS}

Electrostatics is a subset of Maxwell's electrodynamics, valid for time independent electric fields in the absence of magnetic fields. However, both these conditions are frame dependent, and therefore to describe electrostatics we must specify a rest frame. This can be done covariantly by introducing a timelike vector field $\eta^{\mu}$, that is constant and unit normalised:

$$
\partial_{\mu} \eta^{\nu}=0, \quad \eta^{\mu} \eta_{\mu}=+1
$$

Using $\eta^{\mu}$ we can describe the electrostatic field through the 4-potential $A^{\mu}$ :

$$
A^{\mu}=\phi \eta^{\mu}
$$

where $\phi$ is the electrostatic potential. Substituting (2) into Maxwell's equations

$$
\partial_{\mu} F^{\mu \nu}=J^{\nu}, \quad F_{\mu \nu}=\partial_{\mu} A_{\nu}-\partial_{\nu} A_{\mu},
$$

with the requirement that all spatial currents vanish, $J^{\mu}=\rho \eta^{\mu}$, yields precisely Poisson's equation

$$
\nabla^{\mu} \nabla_{\mu} \phi=\rho,
$$

where the spatial derivative is defined by $\nabla_{\mu}=\left(\delta_{\mu}^{\nu}-\eta_{\mu} \eta^{\nu}\right) \partial_{\nu}$ and the negative sign usually appearing in Poisson's equation is accounted for by the signature of the metric tensor.

In addition to (4), the Maxwell equations (3) imply that $\phi$ must be time independent ${ }^{\dagger}, \eta^{\mu} \partial_{\mu} \phi=0$, so that the assumption that the field is static follows directly from the ansatz (2) and the vanishing of spatial currents.

The electrostatic potential $\phi$ is one component of a 4 -vector, but through the frame vector $\eta^{\mu}$ it is also a bona fide scalar field: $\phi=\eta_{\mu} A^{\mu}$. But does it satisfy the expected field equation for a relativistic scalar field? The most natural equation to compare with (4) is the sourced massless Klein-Gordon equation,

$$
\partial^{\mu} \partial_{\mu} \varphi=\varrho,
$$

which is the relativistic generalisation of Poisson's equation. We here use $\varphi$ and $\varrho$ for the field and its source, to distinguish the scalar case from the electrostatic one.

A solution $(\phi, \rho)$ to (4) automatically satisfies (5), though the converse is not true in general. To ensure the two equations are fully equivalent, we must impose the static constraint $\eta^{\mu} \partial_{\mu} \varphi=0$, which automatically accompanies (4). This is due to the scalar nature of $\varrho$, since there is no equivalent to the vanishing of the spatial current.

Of course, there are infinitely many relativistically invariant equations that we could demand $\varphi$ satisfy, just as there are infinitely many generalisations of Maxwell's equations. However, (3) and (5) are the simplest equations compatible with special relativity, and it is interesting that their (electro-)static forms coincide. To test the limits of this equivalence, we turn now to how these fields interact with particles.

\footnotetext{
${ }^{\dagger}$ More precisely, that any time dependence is pure gauge.
} 


\section{PARTICLE MOTION}

An electrically charged particle responds to electromagnetic fields via the Lorentz force,

$$
m \ddot{x}^{\mu}=e F^{\mu}{ }_{\nu} \dot{x}^{\nu}
$$

where $m$ is the mass and $e$ the charge of the particle, and an overdot represents differentiation with respect to proper time. In the electrostatic field (2), this becomes

$$
m \ddot{x}^{\mu}=e\left(\gamma \partial^{\mu} \phi-\dot{\phi} \eta^{\mu}\right),
$$

where $\gamma$ is the particle's Lorentz factor. Since the components of (6) are not all independent, we can project onto the space perpendicular to $\eta^{\mu}$, and consider

$$
\frac{\mathrm{d} \mathbf{p}}{\mathrm{d} t}=-e \nabla \phi
$$

with $\mathbf{p}=m \mathbf{u}$, where $\mathbf{u}=\dot{\mathbf{x}}$ is the celerity ('proper velocity').

The situation for a particle interacting with the scalar field $\varphi$ is altogether more subtle. For a minimally coupled interaction, the equation of motion for a particle of scalar charge $g$ is

$$
\dot{p}_{\mu}=g \partial_{\mu} \varphi, \quad p_{\mu}=(m+g \varphi) \dot{x}_{\mu} .
$$

The second equation in (9) implies that the scalar field contributes to the particle's effective mass. This is related to the mechanism by which the Higgs field gives mass to fermions in the standard model ${ }^{3}$ through the Yukawa couplings ${ }^{\ddagger}$.

In this case, there is no particular advantage to projecting perpendicular to $\eta^{\mu}$; in any frame, we have

$$
\frac{\mathrm{d} \mathbf{p}}{\mathrm{d} t}=-g \gamma^{-1} \nabla \varphi
$$

but now subject to $\mathbf{p}=(m+g \varphi) \mathbf{u}$.

A number of interesting features can be gleaned from (10). The first is that it agrees with (8) only when the particle is at rest relative to $\eta^{\mu}$. In the ultra-relativistic limit $\gamma \gg 1$, the force on the electric charge remains unchanged, whereas that on the scalar charge is strongly suppressed. An even more stark difference emerges when we compare the corresponding accelerations. Since the momentum of the electric charge takes the standard form $^{\S},(8)$ is equivalent to

$$
\frac{\mathrm{d} \mathbf{u}}{\mathrm{d} t}=-\frac{e}{m} \nabla \phi,
$$

while the dynamical mass of the scalar charge converts (10) to

$$
\frac{\mathrm{d} \mathbf{u}}{\mathrm{d} t}=-\gamma^{-1}[\mathbf{1}+\mathbf{u} \otimes \mathbf{u}] \cdot \nabla \ln \left(1+\frac{g \varphi}{m}\right) .
$$

The difference between (11) and (12) could not be more apparent. The additional factor $\gamma$ reducing the force is more than compensated by the tensor $\mathbf{1}+\mathbf{u} \otimes \mathbf{u}$, which contains two factors of $\gamma$, and so acts to enhance and rotate the acceleration; and the logarithm makes it nonlinear in $\varphi$. They agree only in the nonrelativistic limit, when the interaction is very weak, $g \varphi / m \ll 1$.

We end this Section by considering the particle's energy, which by Noether's theorem ${ }^{4}$ should be constant in static fields. Contracting (7) with $\eta^{\mu}$ yields

$$
\mathcal{E}=m \gamma+e \phi=\text { const. }
$$

\footnotetext{
${ }^{\ddagger}$ But it is quite distinct from the Higgs mechanism itself, which gives mass to gauge particles.

$\S$ There is no distinction between kinetic and canonical momentum in electrostatics.
} 
as we would expect. Note that the arbitrary zero of energy is reflected in the freedom to recalibrate the zero of $\phi$.

For the scalar charge, contracting (9) with $\eta^{\mu}$ yields

$$
\mathcal{E}=(m+g \varphi) \gamma=\text { const. }
$$

with total energy being simply the 0-component of momentum (defined with the dynamical mass). In this case, although adding a constant to the energy obviously will not change its status as a conserved quantity, it cannot be achieved by recalibrating the zero of $\varphi$. The latter instead modifies the 'bare mass' $m$. Note that the result (14) allows us to substitute $\gamma^{-1}$ in (10) or (12), replacing an intrinsic ('particle') quantity $\gamma$ with an extrinsic ('field') one, $\varphi$.

\section{CONCLUSIONS}

Gauss's law of electrostatics evolved into Maxwell's theory of electrodynamics, one of the most elegant, and most useful, elements of physical law. However, that is not the only, nor even the most obvious, way the theory could have been developed to incorporate time dependence and relativity. Had Maxwell been driven by purely theoretical ideals, rather than the desire to explain experimental observations, it is quite possible he would instead have proposed a theory based on the relativistic scalar wave equation.

A better appreciation of electrostatics, as well as the statics of scalar fields, can be obtained by comparing field and particle dynamics in the two cases. We have seen that, for a prescribed charge distribution, the same equations are satisfied by electrostatic and static scalar fields, albeit with slightly different requirements for imposing the 'static' criterion. However, the behaviour of charged particles in these fields is not identical. In terms of the forces acting, the difference is simply a suppression by $\gamma$ in the scalar case, and the two forces agree in the nonrelativistic limit. However, in terms of the resulting accelerations, the two interactions behave very differently, especially for ultra-relativistic particles interacting with strong fields. Such conditions are highly relevant to upcoming high power laser facilities, where temporally varying fields must also be accounted for, as must additional factors, such as self-forces and quantum effects. Such considerations are beyond the scope of the present work, and will be addressed elsewhere. ${ }^{5}$

A further close connection between electrodynamics and scalar fields may be observed by analysing Maxwell's theory in 5D Minkowski spacetime, which contains Maxwell's theory along with an aditional scalar field obeying the massless Klein-Gordon equation, as discussed in Appendix A.

\section{APPENDIX A. MAXWELL'S EQUATIONS IN 5D}

In Kaluza-Klein theory, the Einstein-Hilbert action in 5D is decomposed into the 4D Einstein-Hilbert action; plus the Maxwell action for the electromagnetic field, which here corresponds to additional components of the metric tensor; plus a new gravitational scalar field known as the dilaton. In this Appendix, we show that a similar approach to the 5D Maxwell action can be decomposed into the 4D Maxwell action plus the standard action for a massless scalar field. As a prelude, we demonstrate that magnetostatics in 4D space contains 3D electrostatics.

\section{A.1 Magnetostatics in $4 \mathrm{D}$ space}

Magnetostatics is described by the action

$$
\mathcal{S}[\mathbf{A}]=\int\left(\frac{1}{2} \mathbf{B}^{2}+\mathbf{A} \cdot \mathbf{J}\right) \mathrm{d}^{3} x, \quad \mathbf{B}=\nabla \times \mathbf{A} .
$$

The curl operator is defined only in $3 \mathrm{D}$, so to generalise (15) to $4 \mathrm{D}$ we must replace $\mathbf{B}$ with the exterior derivative of $\mathbf{A}$ :

$$
\mathcal{S}[\mathbf{A}]=\int\left(\frac{1}{4} B^{i j} B_{i j}+A_{i} J^{i}\right) \mathrm{d}^{3} x, \quad B_{i j}=\partial_{i} A_{j}-\partial_{j} A_{i},
$$


where $i, j \in\{1,2,3\}$ and, in this section only, indices are raised with the Kronecker delta $\delta^{i j}$.

Introducing the $4 \mathrm{D}$ vector potential $\mathcal{A}_{M}$ and corresponding 4 -field $\mathcal{B}_{M N}=\partial_{M} \mathcal{A}_{N}-\partial_{N} \mathcal{A}_{M}$, and the vector 4-current $\mathcal{J}^{M}(M, N \in\{1,2,3,4\})$, we can promote (16) to 4 dimensions:

$$
\mathcal{S}^{(4)}[\mathcal{A}]=\int\left(\frac{1}{4} \mathcal{B}^{M N} \mathcal{B}_{M N}+\mathcal{A}_{M} \mathcal{J}^{M}\right) \mathrm{d}^{4} x
$$

Identifying $\mathcal{A}_{i}=A_{i}$ and $\mathcal{J}^{i}=J^{i}$, defining $\mathcal{A}_{4}=-\phi$ and $\mathcal{J}^{4}=\rho$, and assuming there is no variation in the $x^{4}$-direction $\left(\partial_{4} \rightarrow 0\right)$, (17) becomes

$$
\mathcal{S}^{(4)}[\mathcal{A}]=L \int\left(\frac{1}{4} B^{i j} B_{i j}+A_{i} J^{i}+\frac{1}{2} \partial^{i} \phi \partial_{i} \phi-\phi \rho\right) \mathrm{d}^{3} x,
$$

where $L$ is the length of the new dimension. In terms of $\mathbf{B}$ and $\mathbf{E}=-\nabla \phi$, this is

$$
\mathcal{S}[\mathbf{A}, \phi]=L^{-1} \mathcal{S}^{(4)}[\mathcal{A}]=\int\left(\frac{1}{2} \mathbf{B}^{2}+\mathbf{A} \cdot \mathbf{J}+\frac{1}{2} \mathbf{E}^{2}-\phi \rho\right) \mathrm{d}^{3} x,
$$

the sum of the actions for magnetostatics and electrostatics ${ }^{\pi}$.

\section{A.2 Electrodynamics in 5D spacetime}

Following the above approach, consider the standard Maxwell action promoted to 5D:

$$
\mathcal{S}^{(5)}[\mathcal{A}]=-\int\left(\frac{1}{4} \mathcal{F}^{M N} \mathcal{F}_{M N}+\mathcal{A}_{M} \mathcal{J}^{M}\right) \mathrm{d}^{5} x, \quad \mathcal{F}_{M N}=\partial_{M} \mathcal{A}_{N}-\partial_{N} \mathcal{A}_{M},
$$

where now $M, N \in\{0,1,2,3,4\}$.

As above, identify the spacetime components of $\mathcal{A}_{M}$ and $\mathcal{J}^{M}$ with the electromagnetic 4-potential and 4current, $\mathcal{A}_{\mu}=A_{\mu}$ and $\mathcal{J}^{\mu}=J^{\mu}(\mu \in\{0,1,2,3\})$, and introduce a new field $\varphi=-\mathcal{A}_{4}$ and source $\varrho=\mathcal{J}^{4}$. Again assuming no variation in the $x^{4}$-direction, (20) becomes

$$
\mathcal{S}[A, \varphi]=L^{-1} \mathcal{S}^{(5)}[\mathcal{A}]=\int\left(-\frac{1}{4} F^{\mu \nu} F_{\mu \nu}-A_{\mu} J^{\mu}+\frac{1}{2} \partial^{\mu} \varphi \partial_{\mu} \varphi+\varphi \varrho\right) \mathrm{d}^{4} x,
$$

which is the sum of the usual actions for the electromagnetic field $F_{\mu \nu}$ sourced by $J^{\mu}$ and a massless relativistic scalar field $\varphi$ sourced by $\varrho$.

An alternative compactification scheme, allowing for periodic variations in the $x^{4}$-direction, introduces a hierarchy of massive fields, while allowing the scalar field to be gauged away. ${ }^{6}$

\section{ACKNOWLEDGMENTS}

We would like to thank David Burton and other members of the ALPHA-X Collaboration for useful discussions. This work was supported by the UK EPSRC (Grant No. EP/N028694/1) and the European Union H2020 Research and Innovation Programme LASERLAB EUROPE (Grant No. 871124). All of the results can be fully reproduced using the methods described in the paper.

ॠA factor (-1) between the electric and magnetic contributions would appear if we took the extra dimension to be timelike, leading to the familiar Maxwell action for electrodynamics. 


\section{REFERENCES}

[1] Kaluza, T., "Zum Unitätsproblem in der Physik," Sitzungsber. Preuss. Akad. Wiss. Berlin (Phys. Math.), 966 (1921).

[2] Bauer, D., Mulser, P., and Steeb, W.-H., "Relativistic ponderomotive force, uphill acceleration, and transition to chaos," Phys. Rev. Lett. 75, 4622 (1995).

[3] Weinberg, S., "A model of leptons," Phys. Rev. Lett. 19, 1264 (1967).

[4] Noether, E., "Invariante Variationsprobleme," Nachr. d. König. Gesellsch. d. Wiss. zu Göttingen, Math-phys. Klasse, 235 (1918).

[5] Noble, A., Burton, D. A., Docherty, L., and Jaroszynski, D. A., "Self-force on a charged particle in an external scalar field," in preparation (2021).

[6] De los Santos, G. and Linares, R., "Canonical analysis of electrodynamics in 5D," AIP Conf. Proc. 1256, 178 (2010). 